\begin{tabular}{|c|l|}
\hline Title & Steady solution and its stability to Navier-Stokes equations with general Navier slip boundary condition \\
\hline Author(s) & Itoh, Shigeharu; Tanaka, Naoto; Tani, A tusi \\
\hline Citation & Hokkaido University Preprint Series in Mathematics, 643, 1-23 \\
\hline Issue Date & 2004 \\
\hline DOI & 10.14943/83796 \\
\hline Doc URL & http://hdl.handle.net/2115/69450 \\
\hline Type & bulletin (article) \\
\hline File Information & pre643.pdf \\
\hline
\end{tabular}

Instructions for use 


\title{
Steady solution and its stability to Navier-Stokes equations with general Navier slip boundary condition
}

\author{
S.Itoh, N.Tanaka and A.Tani \\ Dedicated to the memory of Olga Ladyzenskaya
}

Shigeharu Itoh

Hirosaki University, Department of Mathematics, Hirosaki 036-8560, Japan, sitoh@cc.hirosaki-u.ac.jp

Naoto Tanaka

Fukuoka University, Department of Applied Mathematics, Fukuoka 814-0180, Japan, naoto@cis.fukuoka-u.ac.jp

Atusi Tani

Keio University, Department of Mathematics, Yokohama 223-8522, Japan, tani@math.keio.ac.jp

\begin{abstract}
Steady solution and asymptotic behaviour of corresponding nonsteady solution are studied for the Navier-Stokes equations under general Navier slip boundary condition. It is proved that the existence of a unique stationary solution and that this solution is asymptotically stable under some restrictions of the data.
\end{abstract}

Mathematics Subject Classification (2000).35B40,35Q30,76D03,76D05.

Keywords. incompressible Navier-Stokes equations, gemeral Navier slip boundary condition, steady solution, stability of steady solution.

\section{Introduction}

The motion of an incompressible viscous fluid in a bounded domain $\Omega$ in $\mathbb{R}^{3}$ is described by the Navier-Stokes equations :

$$
\frac{\partial v}{\partial t}+(v \cdot \nabla) v-\nabla \cdot \mathbf{P}(v, p)=f, \quad \nabla \cdot v=0 .
$$


Here $v=v(x, t)$ is the velocity vector field, $p=p(x, t)$ is the pressure, $\mathbf{P}(v, p)=-p \mathbf{I}_{3}+2 \nu \mathbf{D}(v)$ is the stress tensor, $\mathbf{I}_{3}$ is an identity matrix of degree $3, \mathbf{D}(v)$ is the velocity deformation tensor with the elements $D_{i j}=$ $\frac{1}{2}\left(\frac{\partial v_{i}}{\partial x_{j}}+\frac{\partial v_{j}}{\partial x_{i}}\right), \nu>0$ is a constant coefficient of viscosity and $f=f(x, t)$ is a given vector field of external forces.

Equations (1.1) is considered under the initial condition

$$
\left.v\right|_{t=0}=v_{0}(x)
$$

and the boundary conditions

$$
v \cdot n=0, \quad v \cdot \tau=K \mathbf{P} n \cdot \tau,
$$

or equivalently,

$$
v \cdot n=0, \quad v=K[\mathbf{P} n-(\mathbf{P} n \cdot n) n],
$$

where $n$ and $\tau$ are a unit inward normal and a unit tangential vectors to a smooth boundary $\Gamma$ of $\Omega$, respectively, such that $n \times \tau=1$ and $K=K(x, t)$ is assumed to be a nonnegative function defined on $\Gamma_{\infty}=\Gamma \times(0, \infty)$. Dividing both sides of $(1.4)_{2}$ by $1+\nu K$ and using the same letter $K$ in place of $1 /(1+\nu K)$, we have

$$
v \cdot n=0, \quad 2(1-K) \Pi \mathbf{D}(v) n-K v=0,
$$

where $0 \leq K \leq 1$ and $\Pi w=w-(w \cdot n) n$. Condition (1.3) implies that the fluid particles are partially slipping on a solid boundary (see Navier [8], Goldstein [4], Serrin [10], Sect. 64, and references therein ). We note that if $K \equiv 1$, then condition (1.5) reduces the well-investigated adherence one.

The aim of the present paper is to prove the existence of a unique solution $(\bar{v}(x), \bar{p}(x))$ to the stationary problem

$$
\begin{cases}(\bar{v} \cdot \nabla) \bar{v}-\nabla \cdot \mathbf{P}(\bar{v}, \bar{p})=\bar{f}, \quad \nabla \cdot \bar{v}=0 & \text { in } \Omega, \\ \bar{v} \cdot n=0, \quad 2(1-\bar{K}) \Pi \mathbf{D}(\bar{v}) n-\bar{K} \bar{v}=0 & \text { on } \Gamma\end{cases}
$$

and to study its stability with respect to the corresponding nonstationary problem (1.1)-(1.2), (1.5). In $\S \S 3-4$, we prove the following existence theorem to stationary problem (1.6). Throughout Theorems 1.1-1.4 we always assume that the boundary $\Gamma$ belongs to $W_{2}^{\frac{5}{2}+l}\left(\frac{1}{2}<l<1\right)$ (as for function spaces, see $\S 2$ ).

Throughout this paper we denote by $c_{i}(i=1,2,3, \ldots)$ positive constants that will be used later. Otherwise we do not distinguish them and use the same symbol $c$. 
Theorem 1.1 Let $\bar{K} \in W_{2}^{\frac{1}{2}+l}(\Gamma)(0 \leq \bar{K} \leq 1)$. If $\bar{f} \in W_{2}^{l}(\Omega)$ satisfies condition (4.6), then there exists a unique solution $(\bar{v}, \nabla \bar{p}) \in\left(W_{2}^{2+l}(\Omega) \cap\right.$ $H(\Omega)) \times W_{2}^{l}(\Omega)$ of $(1.6)$ satisfying the inequality

$$
\|\bar{v}\|_{W_{2}^{2+l}(\Omega)}+\|\nabla \bar{p}\|_{W_{2}^{l}(\Omega)} \leq c\|\bar{f}\|_{W_{2}^{l}(\Omega)} .
$$

The proof of Theorem 1.1 depends on the investigation of the linearized problem for (1.6) and the contraction mapping principle. In studying the linearized problem we follow the general theory of elliptic boundary value problems developed by $[1,11,16]$. But our problem is not included completely in the framework of known theory $[1,11,16]$, because in boundary condition $2(1-K) \Pi \mathbf{D}(v) n-K v=0$ we must regard both terms $2(1-K) \Pi \mathbf{D}(v) n$ and $K v$ are principal, since $0 \leq K \leq 1$. To overcome this difficulty we make some devices. The most important one is a partition of unity of a domain which was originally introduced by Tani [15] for the study of time-dependent compressible Navier-Stokes equations under condition (1.5), and later used by the authors $[5,14]$ for incompressible case. We follow this idea with some natural modifications for stationary case (see $\S 3.4$ ).

Now let us turn to nonstationary problem (1.1)-(1.2), (1.5). First of all existence of a temporally local solution was established in [14].

Theorem $1.2([14])$ Suppose $K \in W_{2}^{\frac{1}{2}+l, \frac{1}{4}+\frac{l}{2}}\left(\Gamma_{\infty}\right), f \in W_{2}^{l, \frac{l}{2}}\left(Q_{\infty}\right)\left(Q_{\infty}=\right.$ $\Omega \times(0, \infty)), v_{0} \in W_{2}^{1+l}(\Omega)$ and $v_{0}$ satisfies the compatibility conditions. Then problem (1.1)-(1.2), (1.5) has a unique solution $(v, \nabla p) \in W_{2}^{2+l, 1+\frac{l}{2}}\left(Q_{T_{1}}\right) \times$ $W_{2}^{l, \frac{l}{2}}\left(Q_{T_{1}}\right)$ for some $T_{1} \in(0, \infty)$ such that

$$
\|v\|_{W_{2}^{2+l, 1+\frac{l}{2}}\left(Q_{T_{1}}\right)}+\|\nabla p\|_{W_{2}^{l, \frac{l}{2}}\left(Q_{T_{1}}\right)} \leq c_{1}\left(\left\|v_{0}\right\|_{W_{2}^{1+l}(\Omega)}+\|f\|_{W_{2}^{l, \frac{l}{2}}\left(Q_{\infty}\right)}\right) \equiv c_{1} E .
$$

Moreover, the number $T_{1}$ increases unboundedly as $E$ tends to zero.

Theorem 1.2 and the a priori estimates proved in $\S 5$ yield the following temporally global existence theorem.

Theorem 1.3 In addition to the assumptions of Theorem 1.2, suppose $f \in$ $W_{2}^{2 l, l}\left(Q_{\infty}\right)$. If $E_{0}=\left\|v_{0}\right\|_{W_{2}^{1+l}(\Omega)}+\|f\|_{W_{2}^{2 l, l}\left(Q_{\infty}\right)}<\varepsilon_{0}$ with a sufficiently small positive number $\varepsilon_{0}$, then the solution $(v, \nabla p)$ of Theorem 1.2 exists for all $t>0$ and satisfies the inequality

$$
\sup _{t \geq t_{1}>0}\left(\|v(t)\|_{W_{2}^{2+l}(\Omega)}+\|\nabla p(t)\|_{W_{2}^{l}(\Omega)}\right) \leq c E_{0}
$$

for each $t_{1}>0$. 
Finally stability of stationary solution from Theorem 1.1 is discussed in $\S 6$.

Theorem 1.4 Let $(v(x, t), p(x, t))$ and $(\bar{v}(x), \bar{p}(x))$ be the solution of nonstationary problem (1.1)-(1.2), (1.5) from Theorem 1.3 and of stationary problem (1.6) from Theorem 1.1, respectively. Suppose that $\frac{K-\bar{K}}{(1-K)(1-\bar{K})} \in$ $L_{2}(\hat{\Gamma}(t))(\hat{\Gamma}(t)=\{x \in \Gamma \mid K(x, t) \neq 1, \bar{K}(x) \neq 1\})$. Then the difference $u=v-\bar{v}$ obeys the inequality

$$
\|u(t)\|_{L_{2}(\Omega)}^{2} \leq \mathrm{e}^{-M t}\left(\|u(0)\|_{L_{2}(\Omega)}^{2}+\int_{0}^{t} \mathrm{e}^{M s} F(s) \mathrm{d} s\right)
$$

for any $t>0$, where $M$ and $F(t)$ are defined by (6.5) and (6.6), respectively.

In conclution let us mention some previous works about Navier-Stokes and Stokes equations under slip boundary conditions. In the case of perfect slip, i.e., $K \equiv 0$, stationary problem for incompressible Stokes equations was discussed by Solonnikov-Sčadilov [13], while for compressible heat-conductive Navier-Stokes equations by Farwig [2]. Note that the problem with perfect slip condition is closely resemble to that of stationary motion with a free boundary (see e.g., [9]).

On the other hand, the time-dependent problem (1.1)-(1.2), (1.5), besides our previous work [14] mentioned above, was also investigated in [5]. In [5], it was proved that the solution exists for a small time interval in Hölder class of functions and that this solution exists for all time without restriction of smallness of the data provided the space dimension is two. The existence of a temporally global solution for non-homogeneous fluid was also established in $[6]$.

Finally the initial value problem for viscous compressible heat conducting fluid with general slip boundary condition was studied by Tani [15] in Hölder class of functions.

\section{Preliminaries}

\subsection{Function spaces}

Throughout this paper we use the Sobolev-Slobodetskil spaces defined as follows. Let $\Omega$ be a domain in $\mathbb{R}^{n}$ and $l>0$ be a noninteger with an integral part $[l]$ and a nonintegral part $\{l\}$. By $W_{2}^{l}(\Omega)$ we mean the space of functions 
$u(x), x \in \Omega$, equipped with the norm

$$
\begin{aligned}
\|u\|_{W_{2}^{l}(\Omega)}^{2} & =\sum_{|\alpha|=0}^{[l]}\left\|D_{x}^{\alpha} u\right\|_{L_{2}(\Omega)}^{2}+\sum_{|\alpha|=[l]} \int_{\Omega} \int_{\Omega} \frac{\left|D_{x}^{\alpha} u(x)-D_{y}^{\alpha} u(y)\right|^{2}}{|x-y|^{n+2\{l\}}} \mathrm{d} x \mathrm{~d} y \\
& \equiv\|u\|_{W_{2}^{[l]}(\Omega)}^{2}+\|u\|_{\dot{W}_{2}^{l}(\Omega)}^{2},
\end{aligned}
$$

where

$$
D_{x}^{\alpha} u(x)=\frac{\partial^{|\alpha|} u(x)}{\partial x_{1}^{\alpha_{1}} \partial x_{2}^{\alpha_{2}} \cdots \partial x_{n}^{\alpha_{n}}}, \quad \alpha=\left(\alpha_{1}, \alpha_{2}, \ldots, \alpha_{n}\right)
$$

denotes a distributional derivative of $u(x)$ of order $|\alpha|=\alpha_{1}+\alpha_{2}+\cdots+\alpha_{n}$. Now we define anisotropic spaces $W_{2}^{l, \frac{l}{2}}\left(Q_{T}\right)$ of functions $u(x, t)$ in a cylindrical domain $Q_{T}=\Omega \times(0, T)(0<T \leq \infty)$ as $W_{2}^{l, \frac{l}{2}}\left(Q_{T}\right)=L_{2}\left(0, T ; W_{2}^{l}(\Omega)\right) \cap$ $L_{2}\left(\Omega ; W_{2}^{\frac{l}{2}}(0, T)\right)$ and introduce in this space the norm

$$
\begin{aligned}
\|u\|_{W_{2}^{l, \frac{l}{2}}\left(Q_{T}\right)}^{2} & =\int_{0}^{T}\|u(\cdot, t)\|_{W_{2}^{l}(\Omega)}^{2} \mathrm{~d} t+\int_{\Omega}\|u(x, \cdot)\|_{W_{2}^{\frac{l}{2}(0, T)}}^{2} \mathrm{~d} x \\
& \equiv\|u\|_{W_{2}^{l, 0}\left(Q_{T}\right)}^{2}+\|u\|_{W_{2}^{0, \frac{l}{2}}\left(Q_{T}\right)}^{2} \cdot
\end{aligned}
$$

Here the norm in $W_{2}^{\frac{l}{2}}(0, T)$ is defined by

$$
\begin{aligned}
\|u(x, \cdot)\|_{W_{2}^{\frac{l}{2}}(0, T)}^{2}= & \sum_{j=0}^{\left[\frac{l}{2}\right]}\left\|\partial_{t}^{j} u(x, \cdot)\right\|_{L_{2}(0, T)}^{2} \\
& +\int_{0}^{T} \mathrm{~d} t \int_{0}^{t}\left|\partial_{t}^{\left[\frac{l}{2}\right]} u(x, t)-\partial_{t}^{\left[\frac{l}{2}\right]} u(x, t-\tau)\right|^{2} \frac{\mathrm{d} \tau}{\tau^{1+2\left\{\frac{l}{2}\right\}}} \\
\equiv & \|u\|_{W_{2}^{\left[\frac{l}{2}\right]}(0, T)}^{2}+\|u\|_{\dot{W}_{2}^{\frac{l}{2}}(0, T)}^{2},
\end{aligned}
$$

where $\partial_{t}=\frac{\partial}{\partial t}$.

For a smooth manifold $\partial \Omega=\Gamma$, the space $W_{2}^{l}(\Gamma)$ of functions defined on $\Gamma$ is introduced in a standard manner by means of the local coordinates 
and the partition of unity, and $W_{2}^{l, \frac{l}{2}}\left(\Gamma_{T}\right)$ can be defined in the same way as above.

The spaces of vector fields whose components belong to $W_{2}^{l}(\Omega), W_{2}^{l, \frac{l}{2}}\left(Q_{T}\right)$ etc. are denoted by the same notation as the scalar case $W_{2}^{l}(\Omega), W_{2}^{l, \frac{l}{2}}\left(Q_{T}\right)$ etc. and their norms are supposed to be equal to the sum of norms of all its components.

\subsection{Auxiliary lemmas}

In this subsection, we present auxiliary inequalities which will be frequently used in later sections. Hereafter, we assume that $\Omega$ is a bounded domain in $\mathbb{R}^{3}$ with a boundary $\Gamma \in W_{2}^{\frac{5}{2}+l}\left(l>\frac{1}{2}\right)$.

Lemma 2.1 ([7]) Suppose $u \in W_{2}^{1}(\Omega)$. Then $u \in L_{4}(\Gamma)$ and

$$
\|u\|_{L_{4}(\Gamma)} \leq c_{2}\|u\|_{W_{2}^{1}(\Omega)} .
$$

Lemma 2.2 ([3]) Let $u$ be a vector function in $W_{2}^{1}(\Omega)$ satisfying $u \cdot n=0$ on $\Gamma$. Then we have

$$
\|u\|_{L_{2}(\Omega)} \leq c_{3}\|\nabla u\|_{L_{2}(\Omega)} .
$$

Next we state Korn's inequality discussed in [13]. For vectors $u, v \in$ $W_{2}^{1}(\Omega)$, we introduce

$$
E(u, v)=\int_{\Omega} \sum_{i, j=1}^{3}\left(\frac{\partial u_{i}}{\partial x_{j}}+\frac{\partial u_{j}}{\partial x_{i}}\right)\left(\frac{\partial u_{i}}{\partial x_{j}}+\frac{\partial u_{j}}{\partial x_{i}}\right) \mathrm{d} x .
$$

We recall that the vectors satisfying $E(u, u)=0$ form a finite-dimentional affine space of vectors of the form

$$
u=A+B \times x,
$$

where $A$ and $B$ are constant vectors. Let us define $\tilde{H}(\Omega)=\left\{u \in W_{2}^{1}(\Omega) \mid E(u)\right.$ $\equiv E(u, u)<\infty, u \cdot n=0$ on $\Gamma\}$. If $\Omega$ is a region obtained by rotation about a vector $B$, we denote by $H(\Omega)$ the space of functions in $\tilde{H}(\Omega)$ satysfying the condition

$$
\int_{\Omega} u \cdot(B \times x) \mathrm{d} x=0
$$

Otherwise we set $H(\Omega)=\tilde{H}(\Omega)$.

Lemma 2.3 ([13]) The inequality

$$
\|\nabla u\|_{L_{2}(\Omega)}^{2} \leq c_{4} E(u)
$$

is valid for each $u \in H(\Omega)$. 


\section{Stationary Stokes problem}

In this section we consider the problem

$$
\left\{\begin{array}{l}
-\nu \Delta u+\nabla q=\bar{f}, \quad \nabla \cdot u=\rho \quad \text { in } \Omega, \\
2(1-\bar{K}) \Pi \mathbf{D}(u) n-\bar{K} u=b_{\tau}, \quad u \cdot n=b_{n} \quad \text { on } \Gamma .
\end{array}\right.
$$

We prove

Theorem 3.1 Let $\frac{1}{2}<l<1$. Suppose $\Gamma \in W^{\frac{5}{2}+l}, \bar{K} \in W_{2}^{\frac{1}{2}+l}(\Gamma)$,

$$
\left\{\begin{array}{l}
\bar{f} \in W_{2}^{l}(\Omega), \quad \rho \in W_{2}^{1+l}(\Omega), \\
b_{\tau} \in W_{2}^{\frac{1}{2}+l}(\Gamma) \cap W_{2}^{\frac{3}{2}+l}(\gamma), \quad \gamma=\{x \in \Gamma \mid \bar{K}(x)=1\} \\
b_{n} \in W_{2}^{\frac{3}{2}+l}(\Gamma)
\end{array}\right.
$$

and

$$
\int_{\Omega} \rho \mathrm{d} x=-\int_{\Gamma} b_{n} \mathrm{~d} S
$$

Then problem (3.1) has a unique solution $(u, \nabla q)$ such that

$$
(u, \nabla q) \in V_{l} \equiv W_{2}^{2+l}(\Omega) \times W_{2}^{l}(\Omega)
$$

and

$$
\begin{aligned}
& \|(u, \nabla q)\|_{V_{l}} \equiv\|u\|_{W_{2}^{2+l}(\Omega)}+\|\nabla q\|_{W_{2}^{l}(\Omega)} \\
& \leq c_{5}\left(\|\bar{f}\|_{W_{2}^{l}(\Omega)}+\|\rho\|_{W_{2}^{1+l}(\Omega)}+\left\|b_{\tau}\right\|_{W_{2}^{\frac{1}{2}+l}(\Gamma)}+\left\|b_{\tau}\right\|_{W_{2}^{\frac{3}{2}+l}(\gamma)}+\left\|b_{n}\right\|_{W_{2}^{\frac{3}{2}+l}(\Gamma)}\right) \\
& \equiv c_{5}\left\|\left(\bar{f}, \rho, b_{\tau}, b_{n}\right)\right\|_{H_{l}} .
\end{aligned}
$$

As usual we start with the study of a model problem.

\subsection{Half-space problem for the homogeneous system}

First of all, let us consider the boundary value problem for the homogeneous Stokes system in a half space $\mathbb{R}_{+}^{3}=\left\{x=\left(x^{\prime}, x_{3}\right) \mid x^{\prime} \in \mathbb{R}^{2}, x_{3}>0\right\}$ :

$$
\left\{\begin{array}{l}
-\nu \Delta u+\nabla q=0, \quad \nabla \cdot u=0 \quad \text { in } \mathbb{R}_{+}^{3} \\
(1-k)\left(\frac{\partial u_{3}}{\partial x_{j}}+\frac{\partial u_{j}}{\partial x_{3}}\right)-\left.k u_{j}\right|_{x_{3}=0}=b_{j} \quad(j=1,2) \\
\left.u_{3}\right|_{x_{3}=0}=0 \quad \text { on } \mathbb{R}^{2}
\end{array}\right.
$$


where $0 \leq k \leq 1$ is a constant and $b_{1}, b_{2}$ are given functions on $\mathbb{R}^{2}$. Applying to (3.6) the Fourier transform with respect to $x^{\prime}=\left(x_{1}, x_{2}\right)$ :

$$
\hat{f}\left(\xi, x_{3}\right)=F[f]=\int_{\mathbb{R}^{2}} \mathrm{e}^{-i x^{\prime} \cdot \xi} f\left(x^{\prime}, x_{3}\right) \mathrm{d} x^{\prime},
$$

where $\xi=\left(\xi_{1}, \xi_{2}\right), x^{\prime} \cdot \xi=x_{1} \xi_{1}+x_{2} \xi_{2}$. Then we have the following system of ordinary differential equations:

$$
\begin{aligned}
& \left\{\begin{array}{l}
\nu\left(\xi^{2}-\frac{\mathrm{d}^{2}}{\mathrm{~d} x_{3}^{2}}\right) \hat{u}_{j}+i \xi_{j} \hat{q}=0 \quad(j=1,2), \\
\nu\left(\xi^{2}-\frac{\mathrm{d}^{2}}{\mathrm{~d} x_{3}^{2}}\right) \hat{u}_{3}+\frac{\mathrm{d} \hat{q}}{\mathrm{~d} x_{3}}=0 \\
i \xi_{1} \hat{u}_{1}+i \xi_{2} \hat{u}_{2}+\frac{\mathrm{d} \hat{u}_{3}}{\mathrm{~d} x_{3}}=0, \quad(\hat{u}, \hat{q}) \longrightarrow 0 \quad\left(x_{3} \longrightarrow+\infty\right),
\end{array}\right. \\
& \left\{\begin{array}{l}
(1-k)\left(i \xi_{j} \hat{u}_{3}+\frac{\mathrm{d} \hat{u}_{j}}{\mathrm{~d} x_{3}}\right)-\left.k \hat{u}_{j}\right|_{x_{3}=0}=\hat{b}_{j} \quad(j=1,2), \\
\left.\hat{u}_{3}\right|_{x_{3}=0}=0 .
\end{array}\right.
\end{aligned}
$$

We seek the solution to (3.8), (3.9) in the form

$$
\begin{aligned}
\left(\begin{array}{c}
\hat{u}_{1} \\
\hat{u}_{2} \\
\hat{u}_{3} \\
\hat{q}
\end{array}\right)=a_{1}\left[\left(\begin{array}{c}
-|\xi| \\
0 \\
-\left(i \xi_{1}+1\right) \\
-2 \nu|\xi|
\end{array}\right) \mathrm{e}^{-|\xi| x_{3}}+\left(\begin{array}{c}
i \xi_{1} \\
i \xi_{2} \\
-|\xi| \\
0
\end{array}\right) x_{3} \mathrm{e}^{-|\xi| x_{3}}\right] \\
+a_{2}\left[\left(\begin{array}{c}
0 \\
-|\xi| \\
-\left(i \xi_{2}+1\right) \\
-2 \nu|\xi|
\end{array}\right) \mathrm{e}^{-|\xi| x_{3}}+\left(\begin{array}{c}
i \xi_{1} \\
i \xi_{2} \\
-|\xi| \\
0
\end{array}\right) x_{3} \mathrm{e}^{-|\xi| x_{3}}\right] \\
+a_{3}\left(\begin{array}{c}
i \xi_{1} \\
i \xi_{2} \\
-|\xi| \\
0
\end{array}\right) \mathrm{e}^{-|\xi| x_{3}} .
\end{aligned}
$$


The coefficient $\left(a_{1}, a_{2}, a_{3}\right)$ is determined by substituting (3.10) into (3.9). We have

$$
\left(\begin{array}{c}
\hat{u}_{1} \\
\hat{u}_{2} \\
\hat{u}_{3} \\
\hat{q}
\end{array}\right)=\left(\begin{array}{ccc}
G_{11} & G_{12} & 0 \\
G_{21} & G_{22} & 0 \\
0 & 0 & 0 \\
G_{41} & G_{42} & 0
\end{array}\right)\left(\begin{array}{l}
\hat{b}_{1} \\
\hat{b}_{2} \\
0
\end{array}\right) \mathrm{e}^{-|\xi| x_{3}}+\left(\begin{array}{ccc}
H_{11} & H_{12} & 0 \\
H_{21} & H_{22} & 0 \\
H_{31} & H_{32} & 0 \\
0 & 0 & 0
\end{array}\right)\left(\begin{array}{l}
\hat{b}_{1} \\
\hat{b}_{2} \\
0
\end{array}\right) x_{3} \mathrm{e}^{-|\xi| x_{3}},
$$

where

$$
\begin{aligned}
G_{m j} & =-\frac{1}{(1-k)|\xi|+k}\left(\delta_{m j}+\frac{(1-k)}{2(1-k)|\xi|+k} \frac{\left(i \xi_{m}\right)\left(i \xi_{j}\right)}{|\xi|}\right) \quad(m, j=1,2), \\
G_{4 j} & =2 \nu \frac{i \xi_{j}}{2(1-k)|\xi|+k} \quad(j=1,2), \\
H_{m j} & =-\frac{1}{2(1-k)|\xi|+k} \frac{\left(i \xi_{m}\right)\left(i \xi_{j}\right)}{|\xi|} \quad(m, j=1,2), \\
H_{3 j} & =\frac{i \xi_{j}}{2(1-k)|\xi|+k} \quad(j=1,2) .
\end{aligned}
$$

In order to estimate $(3.11)$ in $W_{2}^{l}\left(\mathbb{R}_{+}^{3}\right)$, we make use of Parseval's equality. Indeed, we find

Lemma 3.1 Let us introduce the norms

$$
\begin{aligned}
\|u\|_{l, \mathbb{R}^{2}}^{2} & =\int_{\mathbb{R}^{2}}|\hat{u}(\xi)|^{2}|\xi|^{2 l} \mathrm{~d} \xi \\
\|u\|_{l, \mathbb{R}_{+}^{3}}^{2} & =\sum_{k<l} \int_{\mathbb{R}^{2}}\left\|\left(\frac{\mathrm{d}}{\mathrm{d} x_{3}}\right)^{k} \hat{u}(\xi, \cdot)\right\|_{L_{2}\left(\mathbb{R}_{+}\right)}^{2}|\xi|^{2(l-k)} \mathrm{d} \xi+\int_{\mathbb{R}^{2}}\|\hat{u}(\xi, \cdot)\|_{\dot{W}_{2}^{l}\left(\mathbb{R}_{+}\right)}^{2} \mathrm{~d} \xi .
\end{aligned}
$$

Then the norms $\|u\|_{l, \mathbb{R}^{2}}$ and $\|u\|_{l, \mathbb{R}_{+}^{3}}$ are equivalent to $\|u\|_{\dot{W}_{2}^{l}\left(\mathbb{R}^{2}\right)}$ and $\|u\|_{\dot{W}_{2}^{l}\left(\mathbb{R}_{+}^{3}\right)}$, respectively.

As for the estimates of $\mathrm{e}^{-|\xi| x_{3}}$ and $x_{3} \mathrm{e}^{-|\xi| x_{3}}$, we obtain the following lemma by direct calculations. 
Lemma 3.2 Let $k \geq 0$ be an integer and $\alpha \in(0,1)$. Then we have

$$
\begin{aligned}
& \int_{0}^{\infty}\left|\left(\frac{\mathrm{d}}{\mathrm{d} x_{3}}\right)^{k} \mathrm{e}^{-|\xi| x_{3}}\right|^{2} \mathrm{~d} x_{3} \leq c|\xi|^{2 k-1} \\
& \int_{0}^{\infty} \int_{0}^{\infty}\left|\left(\frac{\mathrm{d}}{\mathrm{d} x_{3}}\right)^{k} \mathrm{e}^{-|\xi|\left(x_{3}+y_{3}\right)}-\left(\frac{\mathrm{d}}{\mathrm{d} x_{3}}\right)^{k} \mathrm{e}^{-|\xi| x_{3}}\right|^{2} \frac{\mathrm{d} x_{3} \mathrm{~d} y_{3}}{y_{3}^{1+2 \alpha}} \leq c|\xi|^{2(k+\alpha)-1} \\
& \int_{0}^{\infty}\left|\left(\frac{\mathrm{d}}{\mathrm{d} x_{3}}\right)^{k} x_{3} \mathrm{e}^{-|\xi| x_{3}}\right|^{2} \mathrm{~d} x_{3} \leq c|\xi|^{2 k-3} \\
& \int_{0}^{\infty} \int_{0}^{\infty}\left|\left(\frac{\mathrm{d}}{\mathrm{d} x_{3}}\right)^{k}\left(x_{3}+y_{3}\right) \mathrm{e}^{-|\xi|\left(x_{3}+y_{3}\right)}-\left(\frac{\mathrm{d}}{\mathrm{d} x_{3}}\right)^{k} x_{3} \mathrm{e}^{-|\xi| x_{3}}\right|^{2} \frac{\mathrm{d} x_{3} \mathrm{~d} y_{3}}{y_{3}^{1+2 \alpha}} \leq c|\xi|^{2(k+\alpha)-3}
\end{aligned}
$$

with a constant $c$ independent of $|\xi|$.

From (3.12) and Lemmas 3.1 and 3.2, we obtain

Lemma 3.3 Let $b=\left(b_{1}, b_{2}\right)=(1-k) b^{N}-k b^{D}$ with $b^{N} \in \dot{W}_{2}^{\frac{1}{2}+l}\left(\mathbb{R}^{2}\right)$, $b^{D} \in \dot{W}_{2}^{\frac{3}{2}+l}\left(\mathbb{R}^{2}\right), l \geq 0$. Then solution (3.11) to problem (3.6) satisfies the inequality

$$
\|u\|_{\dot{W}_{2}^{2+l}\left(\mathbb{R}_{+}^{3}\right)}+\|\nabla q\|_{\dot{W}_{2}^{l}\left(\mathbb{R}_{+}^{3}\right)} \leq c\left(\left\|b^{N}\right\|_{\dot{W}_{2}^{\frac{1}{2}+l}\left(\mathbb{R}^{2}\right)}+\left\|b^{D}\right\|_{\dot{W}_{2}^{\frac{3}{2}+l}\left(\mathbb{R}^{2}\right)}\right) .
$$

\subsection{Non-homogeneous system}

Now we consider the non-homogeneous system

$$
\left\{\begin{array}{l}
-\nu \Delta u+\nabla q=\bar{f}, \quad \nabla \cdot u=\rho \text { in } \mathbb{R}_{+}^{3}, \\
(1-k)\left(\frac{\partial u_{3}}{\partial x_{j}}+\frac{\partial u_{j}}{\partial x_{3}}\right)-\left.k u_{j}\right|_{x_{3}=0}=b_{j} \quad(j=1,2), \\
\left.u_{3}\right|_{x_{3}=0}=b_{3} \text { on } \mathbb{R}^{2} .
\end{array}\right.
$$

We prove

Lemma 3.4 Let $b=\left(b_{1}, b_{2}\right)$ be as in Lemma 3.3. Suppose that $\bar{f} \in \dot{W}_{2}^{l}\left(\mathbb{R}_{+}^{3}\right)$, $\rho \in \dot{W}_{2}^{1+l}\left(\mathbb{R}_{+}^{3}\right), b_{3} \in \dot{W}_{2}^{\frac{3}{2}+l}\left(\mathbb{R}^{2}\right)$ and the condition $\int_{\mathbb{R}_{+}^{3}} \rho \mathrm{d} x=-\int_{\mathbb{R}^{2}} b_{3} \mathrm{~d} x^{\prime}$ is satisfied. Then the solution $(u, \nabla q)$ to problem (3.14) satisfies the estimate

$$
\begin{aligned}
& \|u\|_{\dot{W}_{2}^{2+l}\left(\mathbb{R}_{+}^{3}\right)}+\|\nabla q\|_{\dot{W}_{2}^{l}\left(\mathbb{R}_{+}^{3}\right)} \\
& \leq c\left(\|\bar{f}\|_{\dot{W}_{2}^{l}\left(\mathbb{R}_{+}^{3}\right)}+\|\rho\|_{\dot{W}_{2}^{1+l}\left(\mathbb{R}_{+}^{3}\right)}+\left\|b^{N}\right\|_{\dot{W}_{2}^{\frac{1}{2}+l}\left(\mathbb{R}^{2}\right)}+\left\|b^{D}\right\|_{\dot{W}_{2}^{\frac{3}{2}+l}\left(\mathbb{R}^{2}\right)}+\left\|b_{3}\right\|_{\dot{W}_{2}^{\frac{3}{2}+l}\left(\mathbb{R}^{2}\right)}\right) .
\end{aligned}
$$


Proof. We seek the solution of (3.14) in the form $(u, q)=\left(u^{(1)}+u^{(2)}+\right.$ $\left.u^{(3)}, \nu \rho^{\prime}+q^{(3)}\right)$. Here $u^{(1)}$ is a solution of Dirichlet problem:

$$
-\nu \Delta u^{(1)}=\bar{f} \quad \text { in } \mathbb{R}_{+}^{3},\left.\quad u^{(1)}\right|_{x_{3}=0}=0 \quad \text { on } \mathbb{R}^{2} .
$$

While $u^{(2)}=\nabla \phi$, where $\phi$ is a solution of Neumann problem

$$
\Delta \phi=\rho-\nabla \cdot u^{(1)} \equiv \rho^{\prime} \quad \text { in } \mathbb{R}_{+}^{3},\left.\quad \frac{\partial \phi}{\partial x_{3}}\right|_{x_{3}=0}=b_{3} \quad \text { on } \mathbb{R}^{2},
$$

and $\left(u^{(3)}, q^{(3)}\right)$ is a solution of problem (3.6) with $b=\left(b_{1}, b_{2}\right)$ replaced by $d=\left(d_{1}, d_{2}\right)$, where

$$
\begin{aligned}
d_{j} & =b_{j}-(1-k)\left(\frac{\partial}{\partial x_{j}}\left(u_{3}^{(1)}+u_{3}^{(2)}\right)+\frac{\partial}{\partial x_{3}}\left(u_{j}^{(1)}+u_{j}^{(2)}\right)\right)+\left.k\left(u_{j}^{(1)}+u_{j}^{(2)}\right)\right|_{x_{3}=0} \\
& \equiv(1-k) d_{j}^{N}-k d_{j}^{D} \quad(j=1,2) .
\end{aligned}
$$

From the known estimates to problem (3.16) and (3.17) combined with Lemma 3.3 yields (3.15).

\subsection{Uniqueness of the solution}

Before proving the normal solvability to problem (3.1), we discuss uniqueness of the solution.

Lemma 3.5 The solution to problem (3.1) is unique. (Here uniqueness of $q$ means within an additive constant.)

Proof. Let $(u, q)$ be a solution of (3.1) with $\bar{f}=\rho=b_{\tau}=b_{n}=0$. Then we have

$$
\begin{aligned}
0=-\int_{\Omega}(\nabla \cdot \mathbf{P}(u, q)) \cdot u \mathrm{~d} x & =\int_{\Gamma} \mathbf{P}(u, q) n \cdot u \mathrm{~d} S+\frac{\nu}{2} E(u) \\
& =\int_{\Gamma} 2 \nu \mathbf{D}(u) n \cdot u \mathrm{~d} S+\frac{\nu}{2} E(u) \\
& =\nu \int_{\Gamma^{*}} \frac{\bar{K}}{1-\bar{K}}|u|^{2} \mathrm{~d} S+\frac{\nu}{2} E(u),
\end{aligned}
$$

where $\Gamma^{*}=\{x \in \Gamma \mid \bar{K}(x) \neq 1\}$. Therefore the boundary condition $u \cdot n=0$ implies $u \equiv 0$. 


\subsection{Proof of Theorem 3.1}

Here and in what follows we simply write $\|\cdot\|_{L_{2}(\Omega)}$ as $\|\cdot\|$.

We decompose a solution (3.1) in a similar manner as in $\$ 3.2$. Namely $(u, q)=\left(u^{(1)}+u^{(2)}+v, \nu \rho^{\prime}+p\right)$, where $u^{(1)}, u^{(2)}=\nabla \phi$ and $(v, p)$ satisfy the following equations, respectively:

$$
\begin{aligned}
& -\nu \Delta u^{(1)}=\bar{f} \quad \text { in } \Omega, \quad u^{(1)}=0 \quad \text { on } \Gamma, \\
& -\nu \Delta \phi=\rho-\nabla \cdot u^{(1)} \equiv \rho^{\prime} \quad \text { in } \Omega, \quad \frac{\partial \phi}{\partial n}=b_{n} \quad \text { on } \Gamma, \\
& \left\{\begin{array}{l}
-\nu \Delta v+\nabla p=0, \quad \nabla \cdot v=0 \quad \text { in } \Omega, \\
2(1-\bar{K}) \Pi \mathbf{D}(v) n-\bar{K} v \\
=b_{\tau}-2(1-\bar{K}) \Pi \mathbf{D}\left(u^{(1)}+\nabla \phi\right)+\left.\bar{K}\left(u^{(1)}+\nabla \phi\right)\right|_{\Gamma} \equiv d_{\tau}, \\
v \cdot n=0 \quad \text { on } \Gamma .
\end{array}\right.
\end{aligned}
$$

Since problems (3.18) and (3.19) are well investigated, we have only to consider problem (3.20). The solvability of (3.20) will be proved by the method of the regularizer (cf., $[11,16]$ ), which necessitates to introduce two systems of covering $\left\{\omega^{(k)}\right\}$ and $\left\{\Omega^{(k)}\right\}$ of $\bar{\Omega}$. As was mentioned in introduction, we make some devices for $\left\{\omega^{(k)}\right\}$ and $\left\{\Omega^{(k)}\right\}$ because of boundary condition $2(1-\bar{K}) \Pi \mathbf{D}(v) n-\bar{K} v=d_{\tau}(0 \leq \bar{K} \leq 1)$.

For arbitrary small positive number $\lambda,\left\{\omega^{(k)}\right\}$ and $\left\{\Omega^{(k)}\right\}$ are constructed as follows :

For $k=k^{\prime}$ satisfying $\omega^{\left(k^{\prime}\right)} \cap \Gamma=\emptyset,\left\{\omega^{\left(k^{\prime}\right)}\right\}$ and $\left\{\Omega^{\left(k^{\prime}\right)}\right\}$ are the cubes with the same center and with the length of their edges, in a parallel direction with axes, equal to $\lambda / 2$ and $\lambda$, respectively.

For $k=k^{\prime \prime}$ such that $\xi^{\left(k^{\prime \prime}\right)} \in \Gamma-\gamma(\gamma=\{x \in \Gamma \mid \bar{K}(x)=1\})$, we define by the local rectangular coordinate system $\{y\}$ :

$$
\begin{aligned}
& \omega^{\left(k^{\prime \prime}\right)}=\Pi_{x}^{y}\left\{\left|y_{j}\right| \leq \frac{1}{2} \beta_{1} \lambda \quad(j=1,2), 0 \leq y_{3}-F\left(y^{\prime} ; \xi^{\left(k^{\prime \prime}\right)}\right) \leq \beta_{1} \lambda\right\} \\
& \Omega^{\left(k^{\prime \prime}\right)}=\Pi_{x}^{y}\left\{\left|y_{j}\right| \leq \beta_{1} \lambda(j=1,2), 0 \leq y_{3}-F\left(y^{\prime} ; \xi^{\left(k^{\prime \prime}\right)}\right) \leq 2 \beta_{1} \lambda\right\} .
\end{aligned}
$$

Here the equation $y_{3}=F\left(y^{\prime} ; \xi^{\left(k^{\prime \prime}\right)}\right)\left(y^{\prime}=\left(y_{1}, y_{2}\right)\right)$ represents the boundary $\Gamma$ in the neighborhood of the point $\xi^{\left(k^{\prime \prime}\right)}, \Pi_{x}^{y}$ is the transformation from $\{y\}$ to $\{x\}$ and $\beta_{1}$ is some positive constant independent of $\lambda$. If $\gamma$ is covered by $\cup_{k^{\prime \prime}}\left(\omega^{\left(k^{\prime \prime}\right)} \cap \Gamma\right)$, then it is clear that $\bar{\Omega}$ is covered by $\left\{\omega^{(k)}\right\}$ and $\left\{\Omega^{(k)}\right\}$ constructed above.

Otherwise (in this case we shall denote $\left.k=k^{\prime \prime \prime}\right)$, we define $\left\{\omega^{\left(k^{\prime \prime \prime}\right)}\right\}$ and 
$\left\{\Omega^{\left(k^{\prime \prime \prime}\right)}\right\}$ by the same way as $\left\{\omega^{\left(k^{\prime \prime}\right)}\right\}$ and $\left\{\Omega^{\left(k^{\prime \prime}\right)}\right\}$ with another positive constant $\beta_{2}\left(\leq \beta_{1}\right)$ also independent of $\lambda$ so that $\gamma-\cup_{k^{\prime \prime}}\left(\omega^{\left(k^{\prime \prime}\right)} \cap \Gamma\right) \subset \cup_{k^{\prime \prime \prime}}\left(\Omega^{\left(k^{\prime \prime \prime}\right)} \cap\right.$ Г) $\subset \gamma$.

Once we introduce the system of coverings as above, the rest of the proof is carried out in line with the general theory of Solonnikov [11]. Hence we only describe it briefly.

Now we consider two families of smooth functions $\left\{\zeta^{(k)}(x)\right\}$ and $\left\{\eta^{(k)}(x)\right\}$ associated with the coverings $\left\{\omega^{(k)}\right\}$ and $\left\{\Omega^{(k)}\right\}: \zeta^{(k)}(x)=1$ if $x \in \omega^{(k)}(t)$, $\zeta^{(k)}(x)=0$ if $x \in \bar{\Omega}-\Omega^{(k)}, 0 \leq \zeta^{(k)}(x) \leq 1,\left|D_{x}^{r} \zeta^{(k)}(x)\right| \leq c \lambda^{-|r|}, \eta^{(k)}(x) \equiv$ $\frac{\zeta^{(k)}(x)}{\sum_{k}\left(\zeta^{(k)}(x)\right)^{2}}$. Ovbiously, $\left\{\eta^{(k)}(x)\right\}$ are smooth functions such that $\eta^{(k)}(x)=0$ if $x \in \bar{\Omega}-\Omega^{(k)}, \sum_{k} \eta^{(k)}(x) \zeta^{(k)}(x)=1$ and

$$
\left|D_{x}^{r} \eta^{(k)}(x)\right| \leq c \lambda^{-|r|} .
$$

We note that $\Gamma \in W^{\frac{5}{2}+l}$ means that $F\left(y^{\prime} ; \xi^{(k)}\right) \in W^{\frac{5}{2}+l}\left(B_{r}\right), B_{r} \equiv\left\{y^{\prime} \in\right.$ $\left.\mathbb{R}^{2}|| y^{\prime} \mid<r\right\}$, has the properties $F(0)=0, \nabla^{\prime} F(0)=0,\|F\|_{W^{\frac{5}{2}+l}\left(B_{r}\right)} \leq N$ with the constants $r$ and $N$ being independent of $y^{\prime}$. We take $\lambda$ small enough so that $\beta_{1} \lambda \leq \frac{r}{2}$ holds. Clearly,

$$
\begin{aligned}
& \left|F\left(y^{\prime}\right)\right|=\left|F\left(y^{\prime}\right)-F(0)\right| \leq N\left|y^{\prime}\right|, \\
& \left|\nabla^{\prime} F\left(y^{\prime}\right)\right|=\left|\nabla^{\prime} F\left(y^{\prime}\right)-\nabla^{\prime} F(0)\right| \leq N\left|y^{\prime}\right| .
\end{aligned}
$$

We define operator $\mathcal{R}$ by

$$
\begin{aligned}
\mathcal{R} h & =\sum_{k^{\prime \prime}, k^{\prime \prime \prime}} \eta^{(k)}(x) \Pi_{x}^{z} \mathcal{R}^{(k)} \Pi_{z}^{x}\left(h \zeta^{(k)}\right)(x) \\
& =\sum_{k^{\prime \prime}, k^{\prime \prime \prime}} \eta^{(k)}(x) \Pi_{x}^{z}\left(\bar{v}^{(k)}, \nabla \bar{p}^{(k)}\right)(z) \\
& =\sum_{k^{\prime \prime}, k^{\prime \prime \prime}} \eta^{(k)}(x)\left(v^{(k)}, \nabla p^{(k)}\right)(x)
\end{aligned}
$$

where $h=\left(0,0, d_{\tau}, 0\right)$, the local coordinate system $\{z\}$ connected with $\{y\}$ is given by the relation $z^{\prime}=y^{\prime}, z_{3}=y_{3}-F\left(y^{\prime} ; \xi^{\left(k^{\prime \prime}\right)}\right)$, $\Pi_{x}^{z}$ is the transformation from $\{z\}$ to $\{x\}, \Pi_{z}^{x}$ is its inverse and $\mathcal{R}^{(k)} \Pi_{z}^{x}\left(h \zeta^{(k)}\right)(x)=\left(\bar{v}^{(k)}, \nabla \bar{p}^{(k)}\right)(z)$, $\left(\bar{v}^{(k)}, \nabla \bar{p}^{(k)}\right)(z)$ is the solution of following problem in the half space $\mathbb{R}_{+}^{3(k)}=$ 
$\Pi_{x}^{z} \Omega^{(k)}:$

$\left\{\begin{array}{l}-\nu \Delta \bar{v}^{(k)}+\nabla \bar{p}^{(k)}=0, \quad \nabla \cdot \bar{v}^{(k)}=0 \quad \text { in } \mathbb{R}_{+}^{3(k)}, \\ \left(1-\bar{K}\left(\xi^{(k)}, 0\right)\right)\left(\frac{\partial \bar{v}_{3}^{(k)}}{\partial x_{j}}+\frac{\partial \bar{v}_{j}^{(k)}}{\partial x_{3}}\right)-\left.\bar{K}\left(\xi^{(k)}, 0\right) \bar{v}_{j}^{(k)}\right|_{z_{3}=0}=\Pi_{z}^{x} \zeta^{(k)} d_{\tau j} \quad(j=1,2), \\ \left.\bar{v}_{3}^{(k)}\right|_{z_{3}=0}=0 \quad \text { on } \mathbb{R}^{2(k)}=\Pi_{z}^{x} \partial \Omega^{(k)} \cap\left\{z_{3}=0\right\} .\end{array}\right.$

Then one can easily show that $\mathcal{R} h=\left(v^{\prime}, \nabla p^{\prime}\right)(x)$ satisfies

$$
\left\{\begin{array}{l}
-\nu \Delta v^{\prime}+\nabla p^{\prime}=\mathcal{M}_{1} h, \quad \nabla \cdot v^{\prime}=\mathcal{M}_{2} h \quad \text { in } \Omega \\
2(1-\bar{K}) \Pi \mathbf{D}\left(v^{\prime}\right) n-\bar{K} v^{\prime}=d_{\tau}+\mathcal{M}_{3} h \\
v^{\prime} \cdot n=\mathcal{M}_{4} h \quad \text { on } \Gamma .
\end{array}\right.
$$

Here the operator $\mathcal{M}=\left(\mathcal{M}_{1}, \mathcal{M}_{2}, \mathcal{M}_{3}, \mathcal{M}_{4}\right)$ is defined on $H_{l}=\left\{\left(\bar{f}, \rho, b_{\tau}, b_{n}\right) \mid\right.$ $\left(\bar{f}, \rho, b_{\tau}, b_{n}\right)$ has the smoothness property in (3.2)\} equipped with the norm $\left\|\left(\bar{f}, \rho, b_{\tau}, b_{n}\right)\right\|_{H_{l}}$ (cf.(3.5)), and is represented as follows:

$$
\begin{aligned}
\mathcal{M}_{1} h= & \sum_{k^{\prime \prime}, k^{\prime \prime \prime}}\left(-\nu\left(\Delta\left(\eta^{(k)} v^{(k)}\right)-\eta^{(k)} \Delta v^{(k)}\right)+\nabla\left(\eta^{(k)} p^{(k)}\right)-\eta^{(k)} \nabla p^{(k)}\right) \\
& \quad+\sum_{k^{\prime \prime}, k^{\prime \prime \prime}} \eta^{(k)} \Pi_{x}^{z}\left(-\nu\left(\Delta^{(k)}-\Delta\right) \bar{v}^{(k)}+\left(\nabla^{(k)}-\nabla\right) \bar{p}^{(k)}\right) \\
\equiv & \mathcal{T}_{1} h+\mathcal{K}_{1} h
\end{aligned}
$$

$$
\begin{aligned}
\mathcal{M}_{2} h & =\sum_{k^{\prime \prime}, k^{\prime \prime \prime}}\left(\nabla \cdot\left(\eta^{(k)} v^{(k)}\right)-\eta^{(k)} \nabla \cdot v^{(k)}\right)+\sum_{k^{\prime \prime}, k^{\prime \prime \prime}} \eta^{(k)} \Pi_{x}^{z}\left(\nabla^{(k)}-\nabla\right) \cdot \bar{v}^{(k)} \\
& \equiv \mathcal{T}_{2} h+\mathcal{K}_{2} h
\end{aligned}
$$$$
\begin{aligned}
\mathcal{M}_{3} h=\sum_{k^{\prime \prime}} 2(1-\bar{K}(x)) \Pi\left(\mathbf{D}\left(\eta^{(k)} v^{(k)}\right) n-\eta^{(k)} \mathbf{D}\left(v^{(k)}\right) n\right) \\
+\sum_{k^{\prime \prime}}\left(\eta^{(k)}\left(\bar{K}\left(\xi^{(k)}\right)-\bar{K}(x)\right)\left(2 \Pi \mathbf{D}\left(v^{(k)}\right) n+v^{(k)}\right)\right. \\
\left.\quad+2 \eta^{(k)}\left(1-\bar{K}\left(\xi^{(k)}\right)\right) \Pi_{x}^{z}\left(\Pi^{(k)}\left(\bar{v}^{(k)}\right) n-\Pi_{0} \mathbf{D}\left(\bar{v}^{(k)}\right) n_{0}\right)\right)
\end{aligned}
$$$$
\equiv \mathcal{T}_{3} h+\mathcal{K}_{3} h
$$$$
\mathcal{M}_{4} h=\sum_{k^{\prime \prime}, k^{\prime \prime \prime}} \eta^{(k)} v^{(k)} \cdot\left(n-n_{0}\right) \equiv \mathcal{K}_{4} h
$$ 
where $\nabla^{(k)}=\Pi_{z}^{x} \nabla_{x}={ }^{t}\left(\frac{\partial x_{i}}{\partial z_{j}}\right)^{-1} \nabla_{z} \equiv \mathbf{g} \nabla_{z}, \Delta^{(k)}=\nabla^{(k)} \cdot \nabla^{(k)}, \mathbf{D}^{(k)}=\Pi_{z}^{x} \mathbf{D}$, $n_{0}=n\left(\xi^{(k)}\right)=(0,0,1)^{t}$ and $\Pi_{0} w=w-\left(w \cdot n_{0}\right) n_{0}$.

If we denote by $\mathcal{A}$ the differential operators in the left hand side of (3.23), then we find

$$
\mathcal{A R} h=h+\mathcal{T} h+\mathcal{K} h,
$$

where $\mathcal{T}=\left(\mathcal{T}_{1}, \mathcal{T}_{2}, \mathcal{T}_{3}, 0\right)$ and $\mathcal{K}=\left(\mathcal{K}_{1}, \mathcal{K}_{2}, \mathcal{K}_{3}, \mathcal{K}_{4}\right)$.

To estimate $\|\mathcal{T} h\|_{H_{l}}$ and $\|\mathcal{K} h\|_{H_{l}}$, we introduce the norm depending on parameter $\lambda$ :

$$
\langle u\rangle_{l, \Omega^{(k)}}^{2}=\sum_{j=0}^{[l]} \frac{1}{\lambda^{l-j}}\|u\|_{W_{2}^{j}\left(\Omega^{(k)}\right)}^{2}+\|u\|_{\dot{W}_{2}^{l}\left(\Omega^{(k)}\right)}^{2} .
$$

Certainly the norms $\|u\|_{W_{2}^{l}\left(\Omega^{(k)}\right)}$ and $\langle u\rangle_{l, \Omega^{(k)}}$ are equivalent for each $\lambda>0$, and the interpolation inequality implies

$$
\|u\|_{W_{2}^{m}\left(\Omega^{(k)}\right)} \leq c \lambda^{l-m}\langle u\rangle_{l, \Omega^{(k)}}
$$

for $0 \leq m<l$. By making use of (3.21), (3.22) and the smoothness of $\bar{K}(x)$, one can show that $\mathcal{K}$ is a contraction operator on $H_{l}$ for small $\lambda$, and $\mathcal{T}$ is a compact operator on $H_{l}$ for each $\lambda$, since the imbedding operator from $H_{l}$ into $H_{l-m}$ is compact for a bounded $\Omega$. Therefore, (3.24) implies that existence of the right regularizer.

Now let us consider $\mathcal{R} \mathcal{A} u$. Similar calculations yield

$$
\mathcal{R} \mathcal{A} u=u+\mathcal{S} u+\mathcal{Q} u
$$

where $u=(v, \nabla p) \in V_{l}, \mathcal{S}=\left(\mathcal{S}_{1}, \mathcal{S}_{2}, \mathcal{S}_{3}, 0\right), \quad \mathcal{Q}=\left(\mathcal{Q}_{1}, \mathcal{Q}_{2}, \mathcal{Q}_{3}, \mathcal{Q}_{4}\right)$,

$$
\begin{aligned}
& \mathcal{S}_{1} u=\sum_{k^{\prime \prime}, k^{\prime \prime \prime}} \eta^{(k)} \Pi_{x}^{z} \mathcal{R}^{(k)} \Pi_{z}^{x}\left(-\nu\left(\zeta^{(k)} \Delta v-\Delta\left(\zeta^{(k)} v\right)\right)+\zeta^{(k)} \nabla p-\nabla\left(\zeta^{(k)} p\right)\right), \\
& \mathcal{Q}_{1} u=\sum_{k^{\prime \prime}, k^{\prime \prime \prime}} \eta^{(k)} \Pi_{x}^{z} \mathcal{R}^{(k)}\left(-\nu\left(\Delta^{(k)}-\Delta\right) \bar{v}^{(k)}+\left(\nabla^{(k)}-\nabla\right) \bar{p}^{(k)}\right), \\
& \mathcal{S}_{2} u=\sum_{k^{\prime \prime}, k^{\prime \prime \prime}} \eta^{(k)} \Pi_{x}^{z} \mathcal{R}^{(k)} \Pi_{z}^{x}\left(\zeta^{(k)} \nabla \cdot v-\nabla \cdot\left(\zeta^{(k)} v\right)\right), \\
& \mathcal{Q}_{2} u=\sum_{k^{\prime \prime}, k^{\prime \prime \prime}} \eta^{(k)} \Pi_{x}^{z} \mathcal{R}^{(k)}\left(\nabla^{(k)}-\nabla\right) \cdot \bar{v}^{(k)} \\
& \mathcal{S}_{3} u=\sum_{k^{\prime \prime}} \eta^{(k)} \Pi_{x}^{z} \mathcal{R}^{(k)} \Pi_{z}^{x} 2(1-\bar{K}(x)) \Pi\left(\zeta^{(k)} \mathbf{D}(v) n-\mathbf{D}\left(\zeta^{(k)} v\right) n\right),
\end{aligned}
$$




$$
\begin{aligned}
\mathcal{Q}_{3} u= & \sum_{k^{\prime \prime}} \eta^{(k)} \Pi_{x}^{z} \mathcal{R}^{(k)} \Pi_{z}^{x}\left(\left(\bar{K}\left(\xi^{(k)}\right)-\bar{K}(x)\right)\left(2 \Pi \mathbf{D}\left(\zeta^{(k)} v\right) n+\zeta^{(k)} v\right)\right. \\
& \quad+\sum_{k^{\prime \prime}} \eta^{(k)} \Pi_{x}^{z} \mathcal{R}^{(k)} 2\left(1-\bar{K}\left(\xi^{(k)}\right)\right)\left(\Pi^{(k)}\left(\bar{v}^{(k)}\right) n-\Pi_{0} \mathbf{D}\left(\bar{v}^{(k)}\right) n_{0}\right) \\
\mathcal{Q}_{4} u= & \sum_{k^{\prime \prime}, k^{\prime \prime \prime}} \eta^{(k)} \Pi_{x}^{z} \mathcal{R}^{(k)} \Pi_{z}^{x} \zeta^{(k)} v \cdot\left(n-n_{0}\right) .
\end{aligned}
$$

By exactly the same way as $\mathcal{K}$ and $\mathcal{T}$, one can show that $\mathcal{Q}$ is a contraction operator on $V_{l}$ and that $\mathcal{S}$ is a compact operator on $V_{l}$, which together with (3.25) imply the existence of the left regularizer. By combining these and uniqueness of a solution from Lemma 3.5, Theorem 3.1 is proved.

\section{Proof of Theorem 1.1}

We solve (1.6) by the method of successive approximations. Let $\left(\bar{v}^{(0)}, \nabla \bar{p}^{(0)}\right)=$ $(0,0)$ and $\left(\bar{v}^{(m)}, \nabla \bar{p}^{(m)}\right) \in \bar{X}(\Omega) \equiv\left\{\left(\bar{v}^{(m)}, \nabla \bar{p}^{(m)}\right) \in V_{l} \mid\left\|\left(\bar{v}^{(m)}, \nabla \bar{p}^{(m)}\right)\right\|_{V_{l}} \leq\right.$ $\left.2 c_{5}\|\bar{f}\|_{W_{2}^{l}(\Omega)}\right\}(m=1,2,3, \ldots)$. We define $\left(\bar{v}^{(m+1)}, \nabla \bar{p}^{(m+1)}\right)$ as a solution to the linear problem

$$
\left\{\begin{array}{l}
-\nu \Delta \bar{v}^{(m+1)}+\nabla \bar{p}^{(m+1)}=\bar{f}-\left(\bar{v}^{(m)} \cdot \nabla\right) \bar{v}^{(m)}, \\
\nabla \cdot \bar{v}^{(m+1)}=0 \quad \text { in } \Omega, \\
\bar{v}^{(m+1)} \cdot n=0, \quad 2(1-\bar{K}) \Pi \mathbf{D}\left(\bar{v}^{(m+1)}\right) n-\bar{K} \bar{v}^{(m+1)}=0 \quad \text { on } \Gamma .
\end{array}\right.
$$

By Theorem 3.1 problem (4.1) has a unique solution $\left(\bar{v}^{(m+1)}, \nabla \bar{p}^{(m+1)}\right) \in V_{l}$ satisfying

$$
\begin{aligned}
\left\|\left(\bar{v}^{(m+1)}, \nabla \bar{p}^{(m+1)}\right)\right\|_{V_{l}} & \leq c_{5}\left(\|\bar{f}\|_{W_{2}^{l}(\Omega)}+\left\|\left(\bar{v}^{(m)} \cdot \nabla\right) \bar{v}^{(m)}\right\|_{W_{2}^{l}(\Omega)}\right) \\
& \leq c_{5}\left(\|\bar{f}\|_{W_{2}^{l}(\Omega)}+c_{6}\left\|\bar{v}^{(m)}\right\|_{W_{2}^{1+l}(\Omega)}^{2}\right) \\
& \leq c_{5}\left(\|\bar{f}\|_{W_{2}^{l}(\Omega)}+c_{6}\left(2 c_{5}\|\bar{f}\|_{W_{2}^{l}(\Omega)}\right)^{2}\right) \\
& \leq c_{5}\left(1+4 c_{5}^{2} c_{6}\|\bar{f}\|_{W_{2}^{l}(\Omega)}\right)\|\bar{f}\|_{W_{2}^{l}(\Omega)} .
\end{aligned}
$$

Hence we find $\left(\bar{v}^{(m+1)}, \nabla \bar{p}^{(m+1)}\right) \in \bar{X}(\Omega)$ provided

$$
4 c_{5}^{2} c_{6}\|\bar{f}\|_{W_{2}^{l}(\Omega)} \leq 1
$$


Now let us prove the convergence of the successive approximations. Subtracting from (4.1) the similar equations for $\left(\bar{v}^{(m)}, \nabla \bar{p}^{(m)}\right)$ and setting

$$
\begin{aligned}
& \left(\bar{V}^{(m+1)}, \nabla \bar{P}^{(m+1)}\right)=\left(\bar{v}^{(m+1)}-\bar{v}^{(m)}, \nabla \bar{p}^{(m+1)}-\nabla \bar{p}^{(m)}\right), \text { we obtain } \\
& \left\{\begin{array}{l}
-\nu \Delta \bar{V}^{(m+1)}+\nabla \bar{p}^{(m+1)}=-\left(\bar{v}^{(m)} \cdot \nabla\right) \bar{V}^{(m)}-\left(\bar{V}^{(m)} \cdot \nabla\right) \bar{v}^{(m-1)}, \\
\nabla \cdot \bar{V}^{(m+1)}=0 \quad \text { in } \Omega, \\
\bar{V}^{(m+1)} \cdot n=0, \quad 2(1-\bar{K}) \Pi \mathbf{D}\left(\bar{V}^{(m+1)}\right) n-\bar{K} \bar{V}^{(m+1)}=0 \quad \text { on } \Gamma .
\end{array}\right.
\end{aligned}
$$

By virtue of Theorem 3.1, there exists a unique solution $\left(\bar{V}^{(m+1)}, \nabla \bar{P}^{(m+1)}\right) \in$ $V_{l}$ of (4.4), which satisfies

$$
\begin{aligned}
\left\|\left(\bar{V}^{(m+1)}, \nabla \bar{P}^{(m+1)}\right)\right\|_{V_{l}} & \leq c_{5}\left(\left\|\left(\bar{v}^{(m)} \cdot \nabla\right) \bar{V}^{(m)}\right\|_{W_{2}^{l}(\Omega)}+\left\|\left(\bar{V}^{(m)} \cdot \nabla\right) \bar{v}^{(m-1)}\right\|_{W_{2}^{l}(\Omega)}\right) \\
& \leq c_{5} c_{6}\left(\left\|\bar{v}^{(m)}\right\|_{W_{2}^{1+l}(\Omega)}+\left\|\bar{v}^{(m-1)}\right\|_{W_{2}^{1+l}(\Omega)}\right)\left\|\bar{V}^{(m)}\right\|_{W_{2}^{1+l}(\Omega)} \\
& \leq 4 c_{5}^{2} c_{6}\|\bar{f}\|_{W_{2}^{l}(\Omega)}\left\|\left(\bar{V}^{(m)}, \nabla \bar{P}^{(m)}\right)\right\|_{V_{l}} .
\end{aligned}
$$

Therefore if we assume

$$
4 c_{5}^{2} c_{6}\|\bar{f}\|_{W_{2}^{l}(\Omega)}<1
$$

then we see that the sequence $\left(\bar{v}^{(m)}, \nabla \bar{p}^{(m)}\right)$ converges to some $(\bar{v}, \nabla \bar{p}) \in \bar{X}(\Omega)$ as $m \rightarrow \infty$, which is our desired solution to (1.6). The uniqueness of the solution follows from the estimate similar to (4.5).

\section{$5 \quad$ Proof of Theorem 1.3}

We begin with the conservation of energy for (1.1)-(1.2), (1.5).

Lemma 5.1 The estimate

$$
\|v(t)\| \leq\left\|v_{0}\right\|+\int_{0}^{t}\|f(s)\| \mathrm{d} s \quad(t>0)
$$

is true for the solution $(v, \nabla p)$ to problem (1.1)-(1.2), (1.5).

Proof. Multiplying $v$ for $(1.1)_{1}$ and integrating it over $\Omega$, we have the equality

$$
\frac{1}{2} \frac{\mathrm{d}}{\mathrm{d} t}\|v\|^{2}+\frac{\nu}{2} E(v)=-\int_{\Gamma} \mathbf{P}(v, p) n \cdot v \mathrm{~d} S+\int_{\Omega} f \cdot v \mathrm{~d} x .
$$


Similarly in the proof of Lemma 3.5, we find

$$
\frac{1}{2} \frac{\mathrm{d}}{\mathrm{d} t}\|v\|^{2}+\frac{\nu}{2} E(v)+\nu \int_{\Gamma^{*}(t)} \frac{K}{1-K}|v|^{2} \mathrm{~d} S=\int_{\Omega} f \cdot v \mathrm{~d} x \leq\|f\|\|v\|,
$$

where $\Gamma^{*}(t)=\{x \in \Gamma \mid K(x, t) \neq 1\}$.

For the estimates of higher derivatives of the solution, we follow Solonnikov [12].

Lemma 5.2 Let the solution from Theorem 1.2 satisfy the condition

$$
\|v\|_{W_{2}^{2+l, 1+\frac{l}{2}}\left(Q_{T_{1}}\right)} \leq \delta
$$

with a sufficiently small number $\delta>0$. Then

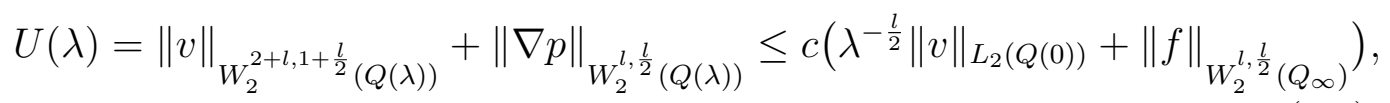

where $\lambda \in(0,1), Q(\lambda)=\Omega \times\left(2 t_{0}+\lambda, T_{1}\right), t_{0}>0,2 t_{0}+\lambda<T_{1}$. Furthermore,

$$
\sup _{t \in\left(t_{1}, T_{1}\right)}\left(\|v(t)\|_{W_{2}^{2+l}(\Omega)}+\|\nabla p(t)\|_{W_{2}^{l}(\Omega)}\right) \leq c\left(\|v\|_{L_{2}(Q(0))}+\|f\|_{W_{2}^{2 l, l}\left(Q_{\infty}\right)}\right)
$$

is valid for each $t_{1} \in\left(2 t_{0}, T_{1}\right)$.

Proof. Let $\zeta_{\lambda}(t)$ be a smooth function of $t \in \mathbb{R}$ which vanishes for $t \leq t_{0}+\frac{\lambda}{2}$, equals to 1 for $t \geq t_{0}+\lambda$ and satisfies $0 \leq \zeta_{\lambda}(t) \leq 1,\left|\zeta_{\lambda}^{(k)}(t)\right| \leq c \lambda^{-k}$. Then it is easily seen that $\left(v_{\lambda}, \nabla p_{\lambda}\right)=\left(\zeta_{\lambda} v, \zeta_{\lambda} \nabla p\right)$ satisfies the equations

$$
\left\{\begin{array}{l}
\frac{\partial v_{\lambda}}{\partial t}-\nabla \cdot \mathbf{P}\left(v_{\lambda}, p_{\lambda}\right)=\zeta_{\lambda} f+\zeta_{\lambda}(v \cdot \nabla) v-\zeta_{\lambda}^{\prime} v, \quad \nabla \cdot v_{\lambda}=0 \quad \text { in } Q(\lambda), \\
\left.v_{\lambda}\right|_{t=0}=0 \quad \text { on } \Omega, \\
v_{\lambda} \cdot n=0, \quad 2(1-K) \Pi \mathbf{D}\left(v_{\lambda}\right) n-K v_{\lambda}=0 \quad \text { on } \Gamma(\lambda)=\Gamma \times\left(2 t_{0}+\lambda, T_{1}\right) .
\end{array}\right.
$$

Applying Theorem 3.4 in [14], we obtain

$$
\begin{aligned}
& \left\|v_{\lambda}\right\|_{W_{2}^{2+l, 1+\frac{l}{2}}\left(Q_{T_{1}}\right)}+\left\|\nabla p_{\lambda}\right\|_{W_{2}^{l, \frac{l}{2}}\left(Q_{T_{1}}\right)} \\
& \leq c\left(\left\|\zeta_{\lambda} f\right\|_{W_{2}^{l, \frac{l}{2}}\left(Q_{T_{1}}\right)}+\left\|\zeta_{\lambda}(v \cdot \nabla) v\right\|_{W_{2}^{l, \frac{l}{2}}\left(Q_{T_{1}}\right)}+\left\|\zeta_{\lambda}^{\prime} v\right\|_{W_{2}^{l, \frac{l}{2}}\left(Q_{T_{1}}\right)}\right) \\
& \leq c\left(\|f\|_{W_{2}^{l, \frac{l}{2}}\left(Q_{T_{1}}\right)}+\delta\left\|v_{\lambda}\right\|_{W_{2}^{1+l, \frac{1}{2}+\frac{l}{2}}\left(Q_{T_{1}}\right)}+\lambda^{-1}\|v\|_{W_{2}^{l, \frac{l}{2}}\left(Q_{T_{1}}\right)}\right) .
\end{aligned}
$$


Therefore,

$$
U(\lambda) \leq c\left(\|f\|_{W_{2}^{l, \frac{l}{2}}\left(Q_{\infty}\right)}+\lambda^{-1}\|v\|_{W_{2}^{l, \frac{l}{2}}\left(Q\left(\frac{\lambda}{2}\right)\right)}\right) .
$$

Using the interpolation inequality (see [12]), we find

$$
c \lambda^{-1}\|v\|_{W_{2}^{l, \frac{l}{2}}\left(Q\left(\frac{\lambda}{2}\right)\right)} \leq \varepsilon_{1}\|v\|_{W_{2}^{2+l, 1+\frac{l}{2}}\left(Q\left(\frac{\lambda}{2}\right)\right)}+\left(\frac{\varepsilon_{1} \lambda}{c}\right)^{-\frac{l}{2}}\|v\|_{L_{2}(Q(0))},
$$

where $\varepsilon_{1}>0$ is an arbitrary small number. Substituting (5.8) into (5.7) leads to

$$
U(\lambda) \leq \varepsilon_{1} U\left(\frac{\lambda}{2}\right)+\left(\frac{\varepsilon_{1} \lambda}{c}\right)^{-\frac{l}{2}}\|v\|_{L_{2}(Q(0))}+c\|f\|_{W_{2}^{l, \frac{l}{2}}\left(Q_{\infty}\right)} .
$$

If $\varepsilon_{1}>0$ is so small that $\frac{\varepsilon_{1}}{2^{\frac{l}{2}}}<1$, then using (5.9) recursively, we get (5.4).

Next consider the difference $\left(v^{(s)}(x, t), \nabla p^{(s)}(x, t)\right)=\left(v_{\lambda}(x, t)-v_{\lambda}(x, t-\right.$ $\left.s), \nabla p_{\lambda}(x, t)-\nabla p_{\lambda}(x, t-s)\right), 0<s<t_{0}$. Subtracting from (5.6) the similar equations for $\left(v_{\lambda}(x, t-s), \nabla p_{\lambda}(x, t-s)\right)$, we obtain the system of equations for $\left(v^{(s)}(x, t), \nabla p^{(s)}(x, t)\right)$. We apply to it Theorem 3.4 in [14] once again. Then the following inequality holds :

$$
\begin{aligned}
& \left\|v^{(s)}\right\|_{W_{2}^{2+l, 1+\frac{l}{2}}\left(Q_{T_{1}}\right)}+\left\|\nabla p^{(s)}\right\|_{W_{2}^{l, \frac{l}{2}}\left(Q_{T_{1}}\right)} \\
& \leq c\left(\left\|\left(\zeta_{\lambda}(v \cdot \nabla) v\right)(t)-\left(\zeta_{\lambda}(v \cdot \nabla) v\right)(t-s)\right\|_{W_{2}^{l, \frac{l}{2}}\left(Q_{T_{1}}\right)}\right. \\
& \left.\quad+\left\|\left(\zeta_{\lambda}^{\prime} v\right)(t)-\left(\zeta_{\lambda}^{\prime} v\right)(t-s)\right\|_{W_{2}^{l, \frac{l}{2}}\left(Q_{T_{1}}\right)}+\left\|\left(\zeta_{\lambda} f\right)(t)-\left(\zeta_{\lambda} f\right)(t-s)\right\|_{W_{2}^{l, \frac{l}{2}}\left(Q_{T_{1}}\right)}\right) .
\end{aligned}
$$

We calculate the right hand side of (5.10), for example, as follows.

$$
\begin{aligned}
& \left\|\left(\zeta_{\lambda}(v \cdot \nabla) v\right)(t)-\left(\zeta_{\lambda}(v \cdot \nabla) v\right)(t-s)\right\|_{W_{2}^{l, \frac{l}{2}}\left(Q_{T_{1}}\right)} \\
& \leq\left\|\left(v^{(s)} \cdot \nabla\right) v(t)\right\|_{W_{2}^{l, \frac{l}{2}}\left(Q_{T_{1}}\right)}+\left\|(v(t-s) \cdot \nabla) v^{(s)}\right\|_{W_{2}^{l, \frac{l}{2}}\left(Q_{T_{1}}\right)} \\
& \quad+\left\|\left(\zeta_{\lambda}(t-s)-\zeta_{\lambda}(t)\right)(v(t-s) \cdot \nabla) v(t)\right\|_{W_{2}^{l, \frac{l}{2}}\left(Q_{T_{1}}\right)} \\
& \leq c\left(\delta\left\|v^{(s)}\right\|_{W_{2}^{1+l, \frac{1}{2}+\frac{l}{2}}\left(Q_{T_{1}}\right)}+\lambda^{-1} \delta s\|v\|_{W_{2}^{1+l, \frac{1}{2}+\frac{l}{2}}(Q(\lambda))}\right),
\end{aligned}
$$




$$
\begin{aligned}
& \left\|\left(\zeta_{\lambda}^{\prime} v\right)(t)-\left(\zeta_{\lambda}^{\prime} v\right)(t-s)\right\|_{W_{2}^{l, \frac{l}{2}}\left(Q_{T_{1}}\right)} \\
& \leq\left\|\left(\zeta_{\lambda}^{\prime}(t)-\zeta_{\lambda}^{\prime}(t-s)\right) v(t)\right\|_{W_{2}^{l, \frac{l}{2}}\left(Q_{T_{1}}\right)}+\left\|\zeta_{\lambda}^{\prime}(t-s)(v(t)-v(t-s))\right\|_{W_{2}^{l, \frac{l}{2}}\left(Q_{T_{1}}\right)} \\
& \leq c\left(\lambda^{-1}+\lambda^{-2}\right) s\|v\|_{W_{2}^{2+l, 1+\frac{l}{2}}(Q(\lambda))} .
\end{aligned}
$$

Therefore we conclude from (5.10) that

$$
\left\|v^{(s)}\right\|_{W_{2}^{2+l, 1+\frac{l}{2}}\left(Q_{T_{1}}\right)}+\left\|\nabla p^{(s)}\right\|_{W_{2}^{l, \frac{l}{2}}\left(Q_{T_{1}}\right)} \leq c\left(\|v\|_{L_{2}(Q(0))}+\|f\|_{W_{2}^{2 l, l}\left(Q_{\infty}\right)}\right) s^{l} .
$$

Since $l>\frac{1}{2}$, (5.11) together with well-known imbedding theorem yields (5.5).

Proof of Theorem 1.3. Let $\varepsilon_{0}$ be a number such that if $E_{0}<\varepsilon_{0}$, then solution $(v, \nabla p)$ from Theorem 1.2 exists on $[0,1]$. This solution satisfies

$$
\|v\|_{W_{2}^{2+l, 1+\frac{l}{2}}\left(Q_{1}\right)}+\|\nabla p\|_{W_{2}^{l, \frac{l}{2}}\left(Q_{1}\right)} \leq c_{1} E_{0},
$$

where $Q_{1}=\Omega \times(0,1)$. Then condition (5.3) is true provided $c_{1} E_{0}<\delta$. On the other hand

$$
\sup _{t \in\left(t_{1}, 1\right)}\|v(\cdot, t)\|_{W_{2}^{2+l}(\Omega)} \leq c_{7} E_{0}
$$

holds by virtue of Lemmas 5.1 and 5.2. Therefore assuming $c_{7} E_{0}<\varepsilon_{0}$, we have

$$
\|v(\cdot, 1)\|_{W_{2}^{2+l}(\Omega)} \leq \varepsilon_{0}
$$

which implies that Theorem 1.2 is applicable for the initial time $t=1$. This means that the solution exists on $[1,2]$. Hence if $E_{0}<\min \left\{\varepsilon_{0}, \frac{\delta}{c_{1}}, \frac{\varepsilon_{0}}{c_{7}}\right\}$, then we can repeat above argument infinitly many times. This completes the proof.

\section{$6 \quad$ Proof of Theorem 1.4}

It is easy to see that $(u, \nabla q)=(v-\bar{v}, \nabla p-\nabla \bar{p})$ satisfies the equations 


$$
\left\{\begin{array}{l}
\frac{\partial u}{\partial t}+(u \cdot \nabla) u-\nabla \cdot \mathbf{P}(u, q)=-((\bar{v} \cdot \nabla) u+(u \cdot \nabla) \bar{v})+(f-\bar{f}), \\
\nabla \cdot u=0 \quad x \in \Omega, t>0, \\
\left.u\right|_{t=0}=v_{0}(x)-\bar{v}(x) \equiv u_{0}(x) \quad x \in \Omega, \\
u \cdot n=0 \\
2(1-\bar{K}) \Pi \mathbf{D}(u) n-\bar{K} u=(K-\bar{K})(2 \Pi \mathbf{D}(v) n+v) \quad x \in \Gamma, t>0 .
\end{array}\right.
$$

Multiplying the first equation of (6.1) by $u$ and integrating it over $\Omega$, we find

$$
\begin{aligned}
& \frac{1}{2} \frac{\mathrm{d}}{\mathrm{d} t}\|u\|^{2}+\frac{\nu}{2} E(u) \\
& =-\int_{\Gamma} \mathbf{P}(u, q) n \cdot u \mathrm{~d} S-\int_{\Omega}(u \cdot \nabla) \bar{v} \cdot u \mathrm{~d} x+\int_{\Omega}(f-\bar{f}) \cdot u \mathrm{~d} x
\end{aligned}
$$

from which

$$
\begin{aligned}
& \frac{1}{2} \frac{\mathrm{d}}{\mathrm{d} t}\|u\|^{2}+\frac{\nu}{2} E(u)+\nu \int_{\Gamma^{*}} \frac{\bar{K}}{1-\bar{K}}|u|^{2} \mathrm{~d} S \\
& =-\nu \int_{\hat{\Gamma}(t)} \frac{K-\bar{K}}{(1-K)(1-\bar{K})} u \cdot v \mathrm{~d} S-\int_{\Omega}(u \cdot \nabla) \bar{v} \cdot u \mathrm{~d} x+\int_{\Omega}(f-\bar{f}) \cdot u \mathrm{~d} x \\
& \leq \nu\left\|\frac{K-\bar{K}}{(1-K)(1-\bar{K})}\right\|_{L_{2}(\hat{\Gamma}(t))}\|u\|_{L_{4}(\Gamma)}\|v\|_{L_{4}(\Gamma)}+\|\nabla \bar{v}\|_{L_{\infty}(\Omega)}\|u\|^{2}+\|f-\bar{f}\|\|u\| \\
& \leq \varepsilon \nu E(u)+\|\nabla \bar{v}\|_{L_{\infty}(\Omega)}\|u\|^{2}+c_{8}\left(\left\|\frac{K-\bar{K}}{(1-\bar{K})(1-K)}\right\|_{L_{2}(\hat{\Gamma}(t))}^{2}\|v\|_{W_{2}^{1}(\Omega)}^{2}+\|f-\bar{f}\|^{2}\right),
\end{aligned}
$$

where $0<\varepsilon<\frac{1}{2}$. Inequality (6.3) can be written as

$$
\frac{\mathrm{d}}{\mathrm{d} t}\|u\|^{2}+M\|u\|^{2} \leq F(t)
$$

with

$$
\begin{gathered}
M=c_{3}^{-2} c_{4}^{-1} \nu(1-2 \varepsilon)-2\|\nabla \bar{v}\|_{L_{\infty}(\Omega)} \\
F(t)=2 c_{8}\left(\left\|\frac{K-\bar{K}}{(1-\bar{K})(1-K)}\right\|_{L_{2}(\hat{\Gamma}(t))}^{2}\|v\|_{W_{2}^{1}(\Omega)}^{2}+\|f-\bar{f}\|^{2}\right) .
\end{gathered}
$$

From (6.4) we can conclude (1.10). 


\section{References}

[1] S. Agmon, A. Douglis and L. Nirenberg, Estimates near the boundary for solutions of elliptic partial differential equations satisfying general boundary conditions I, II. Comm. Pure Appl. Math., 12 (1959), 623727; ibid 17 (1964), 35-92.

[2] R. Farwig, Stationary solutions of compressible Navier-Stokes equations with slip boundary condition. Comm. Partial Differential Equations, 14 (1989), 1579-1606.

[3] G. P. Galdi, An Introduction to the Mathematical Theory of the NavierStokes Equations, Vol.I, Linearized Steady Problems, Springer, Heiderberg, NewYork, (1994).

[4] S. Goldstein, ed., Modern Developments in Fluid Dynamics, Vol. 2, Dover, New York, (1965).

[5] S. Itoh, N. Tanaka and A. Tani, The initial value problem for the NavierStokes equations with general slip boundary condition in Hölder spaces. J. Math. Fluid Mech., 5 (2003), 275-301.

[6] S. Itoh and A. Tani, The initial value problem for the non-homogeneous Navier-Stokes equations with general slip boundary condition. Proc. Roy. Soc. Edinburgh Sect. A, 130 (2000), 827-835.

[7] O. A. Ladyzhenskaya, The Mathematical Theory of Viscous Incompressible Flow. Second English edition, Gordon and Breach, 1969.

[8] C. L. M. H. Navier, M émoire sur les lois du mouvement des fluides. $M$ ém. Acad. Sci. Inst. France, 6 (1823), 389-440.

[9] V. V. Pukhnachov, Plane steady-state problem with a free boundary for the Navier-Stokes equations. Zh. Prikl. Mech. Techn. Fiz., 13, (1972), 91-102.

[10] J. Serrin, Mathematical principles of classical fluid mechanics, in Handbuch der Physik, VIII/1, Springer, 1959, 125-263.

[11] V. A. Solonnikov, General boundary value problems for systems elliptic in the sence of A. Douglis and L. Nirenberg I, II. Amer. Math. Soc. Transl.,(2) 56 (1966), 193-232; Proc. Steklov Inst. Math., 92 (1968), 269-339. 
[12] V. A. Solonnikov, Unsteady motion of a finite mass of fluid, bounded by a free surface. J. Soviet Math., 40 (1988), 672-686.

[13] V. A. Solonnikov and V. E. Sčadilov, On a boundary value problem for a stationary system of Navier-Stokes equations. Proc. Steklov Inst. Math., 125 (1973), 196-210.

[14] A. Tani, S. Itoh and N. Tanaka, The initial value problem for the NavierStokes equations with general slip boundary condition. Adv. Math. Sci. Appl., 4 (1994), 51-69.

[15] A. Tani, The initial value problem for the equations of motion of general fluid with general slip boundary condition. Kôkyuroku, RIMS, Kyoto Univ., 734 (1990), 123-142.

[16] L. R. Volevic, Solvability of boundary value problem for general elliptic systems, Amer. Math. Soc. Transl.,(2) 67 (1968), 182-225. 